\title{
Amplitude Asymmetry: A Direct Link between Ongoing Oscillatory Activity and Event-Related Potentials?
}

\author{
Iris Grothe ${ }^{1 *}$ and Michael Plöchl ${ }^{2 *}$ \\ ${ }^{1}$ Institute for Brain Research, Center for Cognitive Sciences, University of Bremen, D-28334 Bremen, Germany, and ${ }^{2}$ Institute of Cognitive Science, \\ University of Osnabrück, D-49069 Osnabrück, Germany \\ Review of Mazaheri and Jensen (http://www.jneurosci.org/cgi/content/full/28/31/7781)
}

Event-related potentials (ERPs) have been used since the early days of neuroscience to study sensory, perceptual, and cognitive processes. Yet, the electrophysiological phenomena that give rise to ERPs are poorly understood.

ERPs become visible after averaging trials that are aligned to an event (e.g., stimulus onset). Activity that is not time locked to this event will be averaged out, hence the ERP has traditionally been thought of as an additive response, which is induced by the stimulus, riding on top of and being independent from the ongoing background activity. However, modulations of ongoing oscillatory activity have been linked to sensory processes and cognitive processes like attention, memory, and conscious perception (Buzsáki, 2006). Therefore, the assumption that an ERP does not interact with background oscillations is questionable.

Given these drawbacks of the classic "additive" model, another hypothesis has received increasing attention: the "phase-

Received Sept. 30, 2008; revised 0ct. 27, 2008; accepted 0ct. 30, 2008.

I.G. is supported by the BMBF (German Ministry for Education and Research) grant Bernstein Partner-Functional Adaptation of the Visual Cortex.

*I.G. and M.P. contributed equally to this work.

Correspondence should be addressed to either of the following: Iris Grothe, Center for Cognitive Science, Brain Research Institute, P.0. Box 330440, D-28359 Bremen, Germany, E-mail: grothe@brain.uni-bremen.de; or Michael Plöchl, Institut für Kognitionswissenschaft, Universität Osnabrück, Albrechtstrasse 28, D-49076 0snabrück, Germany, E-mail: mploechl@uni-osnabrueck.de.

DOI:10.1523/JNEUROSCI.4670-08.2008

Copyright $\odot 2008$ Society for Neuroscience $\quad 0270-6474 / 08 / 2813025-03 \$ 15.00 / 0$ reset" model states that a stimulus induces (partial) alignment of the phases of ongoing background oscillations. Accordingly, averaging these phase-coherent oscillations over trials results in the ERP. While this approach has the advantage of providing a direct link between ERPs and ongoing oscillations in cognitive processes, some experimental evidence argues against phase resetting as the dominant mechanism for ERP generation (Shah et al., 2004; Mazaheri and Jensen, 2006).

In practice, it has been difficult to test and dissociate the two models. While the additive model predicts a poststimulus increase in power (Fig. 1B) but no phase locking over trials, the opposite is true for the phase-reset model (Fig. 1C). However, power increases induced by an additive component can be masked by a simultaneous power decrease of ongoing activity. On the other hand, adding an evoked component with a certain delay with respect to an event, onto random activity induces phase concentration without a reset of background oscillations. Besides this, ERPs consist of different components, of which latency, amplitude, and frequency may vary. Altogether, this might explain why it has not been possible so far to resolve to what extent each model is capable of explaining ERP generation.

Recently, Mazaheri and Jensen (2008) proposed an alternative mechanism for the generation of ERPs and their magnetoencephalographic counterparts, the event-related fields (ERFs). Their hypoth- esis is based on the assumption that modulation of ongoing alpha activity affects the peaks and the troughs of the alpha activity to different extents. As illustrated in Figure $1 B$ (http://www.jneurosci.org/cgi/ content/full/28/31/7781/F1) of their article, this means that only the amplitudes of the peaks increase or decrease, while the amplitudes of the troughs stay the same (or vice versa). As a result, the mean of the signal before the modulation will be different from the mean after the modulation. In contrast to symmetrically modulated amplitudes, which are averaged out over trials, at least if we assume a random phase distribution over trials [Mazaheri and Jensen (2008), their Fig. 1C (http:// www.jneurosci.org/cgi/content/full/28/ $31 / 7781 / F 1)]$, this difference in the mean of the signal before and after the power modulation will appear as an increase or decrease of the ERF amplitude [Mazaheri and Jensen (2008), their Fig. $1 D$ (http:// www.jneurosci.org/cgi/content/full/28/ 31/7781/F1)].

To investigate this hypothesis, the authors recorded ongoing alpha activity with MEG during rest (eyes closed) and created a measure to quantify the hypothesized asymmetry of amplitude fluctuations $\left(\mathrm{AFA}_{\text {index }}\right)$. The $\mathrm{AFA}_{\text {index }}$ compares the variance of the peaks with the variance of the troughs by taking the normalized difference between the two measures. Accordingly, positive $\mathrm{AFA}_{\text {index }}$ values indicate a stronger modulation of the peaks and negative values indicate a stronger 
modulation of the troughs, while values close to zero indicate symmetrical modulation. Using this measure, Mazaheri and Jensen (2008) were able to show that in ongoing alpha activity the peaks and the troughs are indeed modulated differently. This confirms earlier findings of Nikulin et al. (2007), who also reported asymmetric alpha amplitude modulation using a similar experimental setting. Nikulin et al. (2007) showed in a second experiment that late somatosensory evoked field (SEF) components coincided in time and space with the stimulus induced alpha power change. However, Mazaheri and Jensen (2008) go beyond a mere visual inspection of the overlap between evoked fields and alpha power changes.

The idea that amplitude asymmetry contributes to ERF generation implies that the larger the change in power of alpha oscillations after stimulus onset is, the more the ERF amplitude will deflect from baseline level. Note, however, that a positive ERF deflection could be produced by a power increase while the peaks are modulated [Mazaheri and Jensen (2008), their supplemental Fig. S5 (right) (http://www. jneurosci.org/content/vol28/issue31/ images/data/7781/DC1/Figure_s5.gif)], as well as by a power decrease while the troughs are modulated [Mazaheri and Jensen (2008), their supplemental Fig. S6 (left) (http://www.jneurosci.org/ content/vol28/issue31/images/data/ 7781/DC1/Figure_s6.gif)]. Thus, whether the amplitude of the ERF increases or decreases is not only determined by the power change, but also by the direction of the asymmetry.

To test whether the $\mathrm{AFA}_{\text {index }}$ signs measured during the resting condition would match the predicted relation between ERF amplitudes and alpha power, Mazaheri and Jensen (2008) performed a second experiment. They recorded ongoing oscillatory activity while the subjects were visually stimulated with eccentrically presented gratings, unrelated to the task. Subsequently, they sorted the trials according to baseline-corrected poststimulus alpha power into three bins and calculated the ERF for each bin. Note that the authors refer to baselinecorrected poststimulus power as "power modulation" $\left(\mathrm{P}_{\text {mod }}\right)$, which we think might be misleading, because the amount of baseline corrected poststimulus power can vary regardless of the size of the power change.

If the model holds, recording sites at which the amplitude of the ERF increases with higher poststimulus power should

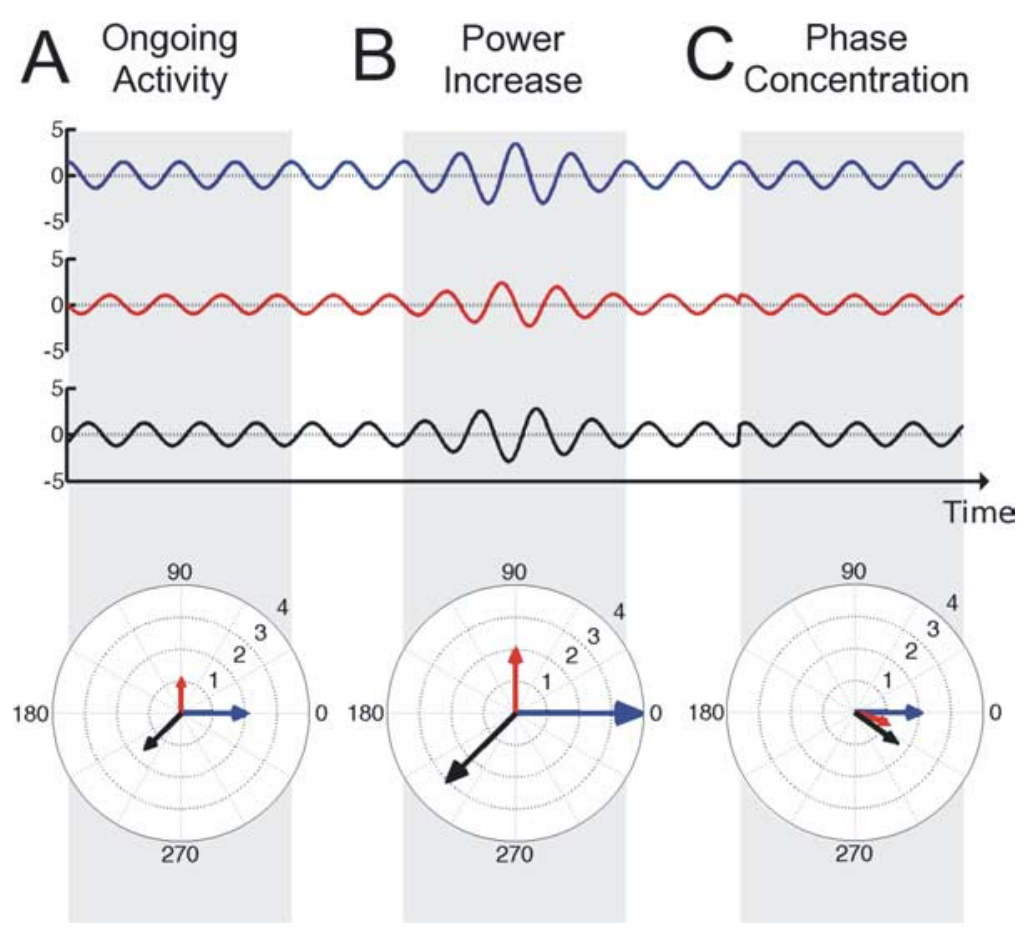

Figure 1. Illustration of power and phase concentration. $\boldsymbol{A}$, Three signals (or trials) with different amplitudes and phases. Power (squared amplitude) and phase of a signal can also be expressed as a vector in a polar coordinate system, where the length of the vector denotes the power of the signal and the angle denotes the phase. $\boldsymbol{B}$, When the amplitude of the signal increases, the power contained in the signal and thus the length of the respective vector increases as well. $\boldsymbol{C}$, When the phases of different signals become more aligned, their respective vectors cluster in one direction. An additive component adds power to the signal, while a reset of the ongoing phase induces phase concentration. Therefore, power and phase concentration traditionally have been assumed to dissociate the additive and the phase reset model. However, in practice this assumption is problematic (see text).

overlap with the sites displaying a positive $\mathrm{AFA}_{\text {index }}$ [Mazaheri and Jensen (2008), their supplemental Figs. S5 $A, D$ (right) (http://www.jneurosci.org/content/ vol28/issue31/images/data/7781/DC1/ Figure_s5.gif), S6A,D (right) (http:// www.jneurosci.org/content/vol28/issue31/ images/data/7781/DC1/Figure_s6.gif)]. Conversely, the sites at which ERF amplitude decreases with higher poststimulus power should display a negative $\mathrm{AFA}_{\text {index }}$ [Mazaheri and Jensen (2008), their supplemental Figs. S5A,D (left) (http://www. jneurosci.org/content/vol28/issue31/ images/data/7781/DC1/Figure_s5.gif), S6A,D (left) (http://www.jneurosci.org/ content/vol28/issue31/images/data/7781/ DC1/Figure_s6.gif)].

The experimental results [Mazaheri and Jensen (2008), their Fig. 4 (http://www. jneurosci.org/cgi/content/full/28/31/7781/ F4)]] indeed show a high correlation between the ERF amplitude and the baseline corrected poststimulus alpha power. Statistical tests confirm that the sign and magnitude of the ERF-alpha power relationship during the visual stimulation task is correlated with the asymmetrical amplitude fluctuations observed during the eyes closed condition [Mazaheri and Jensen (2008), their Fig. 5 (http://www.jneurosci.org/cgi/ content/full/28/31/7781/F5)].

The findings of Mazaheri and Jensen (2008) convincingly demonstrate not only the presence of asymmetric amplitude modulations of alpha activity, but also that this asymmetry contributes to generation of late ERF components. However, a direct connection between amplitude asymmetry and cognitive tasks has not been shown. First, the relation between amplitude fluctuations and the ERF has only been demonstrated for sensory stimulation. Second, the asymmetry of alpha amplitude was computed during a separate resting condition, instead of using the baseline just before stimulation.

A plausible methodological reason for the separate resting condition could be that due to the low power of alpha oscillations in an eyes-opened condition, the signal-to-noise ratio is too small to reliably detect asymmetric modulations. Using a nonvisual modality for stimulation while the subject has its eyes closed might overcome this disadvantage. Another reason for using a separate resting condition could be that, to record sufficiently long segments of alpha activity, each trial would require a baseline period of several 
seconds, resulting in an undesirably long experimental duration. Using prominent and stable ERF components, like the P300, (a positive deflection of the ERF, typically observed $\sim 300$ ms after a subject is presented with an unexpected event) could help to reduce the number of trials, so that the baseline period could be extended while keeping experiment time within reasonable limits.

The results of Mazaheri and Jensen (2008) support the notion that ERFs are directly linked to ongoing activity and hence to the sensory and cognitive functions associated with to oscillatory activity. As the authors note, the amplitude asymmetry is hypothesized to only account for the slow, late components of the ERF. This raises the question of how the amplitude asymmetry hypothesis interrelates with other proposed mechanisms. In line with Fell et al. (2004) and Mazaheri and Jensen (2008) we propose that different mechanisms might be active at different time points. A possible scenario would be that early components are dominantly generated by an additive component, while ongoing activity might influence later components more, when feedback and top-down influences become increasingly important. Extending on this idea, the significant contribution of ongoing activity to ERF generation might increase with higher processing areas.

Findings of Shah et al. (2004) support this proposal. They showed with extracellular electrode measurements in a macaque monkey the occurrence of trial based additive components, but also showed that more prestimulus oscillatory activity is present in the inferior temporal cortex (IT), the highest region of the ventral visual processing hierarchy, than in the primary visual cortex (V1), indicating a higher possibility for top-down or feedback processes to feed in.

In summary, the findings of Mazaheri and Jensen (2008) will not settle the debate about ERF generation, but they clearly have the potential to give it a new impulse and direction. Especially two issues will be of interest for future research: one is to show the direct relation between ERF amplitude and asymmetrical modulation of alpha power during cognition. The other is to investigate how the fast, early components can be explained and how different mechanisms like the additive model and amplitude asymmetry interact. The $\mathrm{AFA}_{\text {index }}$ as a simple and intuitive measure might serve as an easy-toapply tool in this endeavor.

\section{References}

Buzsáki G (2006) Rhythms of the brain. New York: Oxford UP.

Fell J, Dietl T, Grunwald T, Kurthen M, Klaver P, Trautner P, Schaller C, Elger CE, Fernández G (2004) Neural bases of cognitive ERPs: more than phase reset. J Cogn Neurosci 16:1595-1604.

Mazaheri A, Jensen O (2006) Posterior alpha activity is not phase-reset by visual stimuli. Proc Natl Acad Sci U S A 103:2948-2952.

Mazaheri A, Jensen O (2008) Asymmetric amplitude modulations of brain oscillations generate slow evoked responses. J Neurosci 28:7781-7787.

Nikulin VV, Linkenkaer-Hansen K, Nolte G, Lemm S, Müller KR, Ilmoniemi RJ, Curio G (2007) A novel mechanism for evoked responses in the human brain. Eur J Neurosci 25:3146-3154.

Shah AS, Bressler SL, Knuth KH, Ding M, Mehta AD, Ulbert I, Schroeder CE (2004) Neural dynamics and the fundamental mechanisms of event-related brain potentials. Cereb Cortex 14:476-483. 